\title{
Tracking COVID Vaccine Mandates, Outcomes, and Case Rates: Leveraging Informatics to Support the COVID Response
}

\author{
Vrushab Gowda ${ }^{1}$. Jesse M. Ehrenfeld ${ }^{2}$ \\ Published online: 28 August 2021 \\ (c) The Author(s), under exclusive licence to Springer Science+Business Media, LLC, part of Springer Nature 2021
}

\section{Introduction}

The ongoing pandemic (COVID-19) has prompted a fresh wave of clinical informatics tools deployed in the fight against the novel coronavirus. They span a range of functions, including triage applications, screening protocols, and exposure notification programs, many of which have been well-characterized in the literature and media alike. However, parallel efforts to apply informatics tools to a policy tracking context have as yet received scant attention. These would effectively "track the trackers," enabling comparison between seemingly disparate interventions and imposing a sense of methodological order across the potpourri of COVID-19 laws, rules, and guidance across the United States. This editorial discusses the practical utility of developing these informatics and data visualization tools, before outlining a framework for doing so. They stand to offer key decisionmakers an implement for formulating targeted policy and augmenting existing initiatives, and as such should be actively promoted across the healthcare space.

\section{Measuring success and failure}

There is an important opportunity to create systems that can present, at a glance, actionable metrics relevant to policymakers, providers, administrators, health service researchers, and advocates. Much of the existing health policy infrastructure places significant emphasis on aggregate measures such

This article is part of the Topical Collection on Editorials

Jesse M. Ehrenfeld

jehrenfeld@mcw.edu

1 University of North Carolina School of Medicine, Chapel Hill, NC, USA

2 Medical College of Wisconsin, 8701 W Watertown Plank Road, Milwaukee, WI 53226, USA as bed occupancy, mortality and vaccination rates. While important endpoints, these monochromatic figures fail to capture nuances in the public health response to COVID19. Health policy tracking systems can permit a balanced assessment of interventions across the United States in what has been tantamount to a natural experiment in health policy initiatives. This can enable evaluation of rates of adoption, success, and failure, which can in turn be leveraged to inform the design of future interventions. Three case studies do just that, and warrant mentioning here.

\section{Hospital vaccine mandate tracker}

A recent initiative by the Brown School of Public Health provides an illustrative example [1]. In what has become a careful balancing act between public health promotion and sensitive labor relations, healthcare organizations remain divided over requiring COVID vaccinations for their workers in spite of calls for vaccine mandates by the American Medical Association, American Hospital Association, the American Nurses Association and dozens of other professional societies. Brown researchers relied upon an opensource platform (collecting submissions via Google Forms) to record this phenomenon through an encyclopedic listing of employee vaccine mandates at hospitals nationwide. After having effectively crowdsourced data collection, they then stratified results by geography, scope (e.g. whether the mandate applies to the entire health system or select constituent hospitals), and the date the policy was announced. It is moreover freely available online, thereby offering the public at large a readily accessible product.

\section{Resource management and COVID outcomes}

A multidisciplinary team from Harvard Medical School, National Center for Health Research, and analytics consultancy Grinformatics sought to provide a high-level analysis of interstate variation in COVID outcomes relatively early 
on during the pandemic [2]. They interrogated relationships between state caseload and deaths with their respective densities of physicians and ventilators, having controlled for stay-at-home orders. This was then depicted on choropleth maps for vivid comparison; with their graphic representation, the team ranked among the first to do so in the setting of COVID policy interventions.

\section{COVID-19 hospital impact model for epidemics (CHIME)}

Penn Medicine's Predictive Healthcare group has created CHIME, an open-source tool for institutional clients to forecast admissions, capacity, and resource intensity [3]. Unlike the two previously mentioned studies, it is an interactive tool in the fullest sense of the term, allowing users to specify both hospital- and community-level characteristics as input. The development of CHIME was inherently a multidisciplinary effort; it not only relies upon sophisticated epidemiological models, but can moreover interface with geographic information systems (GIS) to represent trends across both space and time.

\section{Informatics in the age of COVID: The policymaker's dashboard}

Future informatics tools should derive inspiration from these programs, adopting their open-source, freely accessible, highly visual, and interactive characteristics to drive high-quality policy. We have identified four domains within the COVID space which would particularly stand to benefit from their deployment: (1) employee vaccine mandates, not merely among healthcare facilities but across sectors and verticals, (2) hospital resource availability, (3) public orders in states, counties, and municipalities, and (4) health outcomes among vulnerable groups, stratified by age, sex, sexual orientation, gender identity, socioeconomic status, race and ethnicity. All feature a heterogenous population subject to range of policies with varying effect; tracking systems would facilitate both side-to-side comparison as well as guide targeted interventions.

This begs three questions: who should develop these tools, how should they be validated, and what comes of the information they yield? Maintaining a compendium is one matter, but driving it into operational policy something altogether different. This highlights the centrality of audience, venue, and the developer-policymaker nexus. Public sector entities such as the Centers for Medicare and Medicare Services (CMS) and the Centers for Disease Control and Prevention (CDC) are well-positioned to create, or at least sponsor these products at the federal level, as are state departments of health within their jurisdictions. As cornerstones of the public health apparatus, all bear considerable institutional legitimacy and offer highly visible platforms. These agencies can and should lead the charge in developing policy informatics applications; they may be tooled for both internal planning (e.g. pandemic wargaming, resource forecasting) and public consumption.

Alternatively, academicians can develop these models in conjunction with thinktanks, nonprofit groups, or professional societies, as in the case of the Dartmouth Atlas of Health Care. This would ensure that individual researchers' output enters a broad audience of healthcare principals, thereby achieving a force multiplier effect. They can moreover interface with public sector entities, such as the aforementioned agencies, to broadcast their capabilities to a wider national audience.

\section{Conclusion}

In many ways, COVID-19 has effaced traditional distinctions between class, race, age, political affiliation, and geography. Discipline does not - and should not - prove the exception. The exigencies of the pandemic demand creative, collaborative solutions bringing together innovative concepts across spheres previously thought far afield. Melding informatics with policy analysis aims to do precisely that. It deserves further scholarly attention, governmental support, and operational use commensurate with its vital importance in the fight against COVID.

\section{References}

1. Renton, B., Nair-Desai, S., Jha, A. K., New: Hospital Vaccine Mandate Tracker. Global Epidemics. 2021. available at: https:// globalepidemics.org/2021/07/24/new-hospital-vaccine-mandatetracker/. Accessed 27 Aug 2021.

2. Ronquillo, J. G., Lester, W. T., Zuckerman, D. M., Using informatics to guide public health policy during the COVID-19 pandemic in the USA. J. Public Health (Oxf). 42(4):660-664, 2020.

3. Becker, B., Chivers, C., Announcing CHIME, A tool for COVID19 capacity planning. Penn Medicine Predictive Healthcare. 2020. available at: http://predictivehealthcare.pennmedicine.org/2020/ 03/14/accouncing-chime.html. Accessed 27 Aug 2021.

Publisher's Note Springer Nature remains neutral with regard to jurisdictional claims in published maps and institutional affiliations. 